\title{
Development and Clinical Use of an Artificial Lung
}

\author{
Nathan L. Kister • Brittany A. Zwischenberger • \\ Jeremiah T. Martin · Joseph B. Zwischenberger
}

Published online: 20 September 2014

(C) Springer Science + Business Media New York 2014

\begin{abstract}
In the 1970s, the use of cardiopulmonary bypass at the bedside for critically ill patients with respiratory failure began and was termed extracorporeal membrane oxygenation (ECMO). Later, in the 1980s, applications for extracorporeal technology expanded, and included oxygenation, $\mathrm{CO}_{2}$ removal, and hemodynamic support. However, early studies regarding the use of ECMO for acute lung failure provided less than optimistic results. Today, recent research has created a renewed interest in such technology. There have been progressive advancements in artificial lung technology, and ECMO serves as a form of life support and as a bridge to transplantation for critically ill patients when traditional supportive care is no longer effective. These progressive advancements in artificial lung technology provide another tool in the critical care physician's arsenal to combat this often fatal injury.
\end{abstract}

Keywords Artificial lung - Extracorporeal membrane oxygenation $\cdot$ Respiratory failure $\cdot$ Transplantation . Ventilation

\section{Introduction}

Despite the continued evolution and advancements in critical care medicine, acute respiratory failure continues to cause

This article is part of the Topical Collection on Artificial Organ CT Surgery.

N. L. Kister · B. A. Zwischenberger - J. T. Martin ·

J. B. Zwischenberger $(\bowtie)$

Division of Cardiothoracic Surgery, Department of Surgery,

University of Kentucky, 800 Rose Street, MN260, Lexington,

KY 40536-0298, USA

e-mail: joseph.zwischenberger@uky.edu significant morbidity and mortality worldwide. Vasilyev et al. [1] found in an intercontinental trial that the in-hospital mortality rates for acute lung injury approached $45 \%$. While positive pressure ventilation has been the cornerstone of managing patients with significant lung injury, there have been progressive advancements in artificial lung technology which may provide another tool in the critical care physician's arsenal to combat this often fatal injury.

Early studies regarding the use of extracorporeal membrane oxygenation (ECMO) for acute lung failure provided less than optimistic results [2]. Recent research, however, has created a renewed interest in such technology. Studies such as the CESAR trial and recent experience with H1N1 pandemics have shown the potential benefit and possible superiority of ECMO to traditional ventilator support [3•, 4]. This article aims to review the development, current use, and potential future direction of artificial lung technology in the adult patient with lung failure.

The term "artificial lung" has for the most part been used to refer to venovenous ECMO (VV-ECMO) although some other support devices which function to assist or replace the oxygenation and ventilation functions of the lung may be grouped under this terminology [5*•]. ECMO is a broad term that encompasses many different variations of extracorporeal oxygenation. The two main types of ECMO are venoarterial (VA) and venovenous (VV). VA-ECMO is most commonly used for patients with both heart and lung failure and functions more as the traditional cardiopulmonary bypass machine in which oxygenated and pressurized blood is returned to the systemic circulation. VV-ECMO more appropriately functions as an artificial lung as it replaces the respiratory component of the lung but does not replace the patient's intrinsic cardiac function. Hence the discussion of this article will focus on the clinical aspects of VV-ECMO rather than the complete cardiopulmonary support of VA-ECMO. 
The need for artificial lung technology to replace the respiratory functions of the lungs (oxygenation and ventilation) is usually secondary to acute lung injury which may be induced by any variety of pulmonary insults, including surgery, trauma, or infection. Only patients whose lung function is expected to make a recovery should be considered candidates for VV-ECMO. Recent advances in technology which allow for patients to be maintained on ECMO for longer periods of time are now questioning whether the artificial lung can be used as a bridge to transplant in patients with end-stage lung disease until donor organs become available [6].

In patients with acute lung injury, mortality can range anywhere from 34 to $58 \%$ [3•]. The vast majority of patients with acute respiratory failure are managed with positive pressure ventilation. Ventilators alone or in combination with other rescue strategies are often able to provide support in the form of oxygenation and ventilation but only if the lung parenchyma itself is not too severely damaged to perform these basic functions. Unfortunately, positive pressure ventilation comes with risks including pneumonia, need for sedation and immobility, and further damage to the lung parenchyma itself through barotrauma, which can escalate the injury to an already compromised lung tissue. VV-ECMO has shown promise in replacing the respiratory function of the lung, while at the same time allowing the native lung tissue to heal without the harsh and unnatural positive pressure ventilation.

For patients who have reached end-stage lung function, transplantation is often the only long-term solution. Since James Hardy performed the first lung transplant in 1963, there has been tremendous progress made related to transplant mortality, short-term survival, and patient quality of life. While our technical and medical advances have meant better outcomes for those patients who are able to receive lung transplant, we have been limited by the number of available donor lungs. With the limited supply and high demand of lung donor availability, the Lung Allocation Score (LAS) has attempted to factor the urgency of individuals on the waiting list into the allocation process, thereby increasing efficiency. Unfortunately, it is not uncommon for a person to be on the waiting list for 2-3 years [7]. In the current era, VV-ECMO may be able to serve as a bridge in which an artificial device can supplement the patient's lung function until the required donor lungs become available [7].

\section{Origins}

The term "artificial lung" refers to a mechanical device which can take over or supplement the respiratory functions of oxygenation and ventilation normally carried out by our native lungs. While our lungs participate in many other physiologic processes including blood filtration, immune defense, provide a cardiac volume reservoir, and participate in many metabolic functions, the focus of the artificial lung as it pertains to this article and VV-ECMO will focus on the more basic pulmonary functions of oxygen delivery and carbon dioxide extraction [8].

\section{ECMO Development}

ECMO had its origin in cardiopulmonary bypass which revolutionized cardiac surgery. In 1953, Gibbon first used artificial oxygenation and perfusion support for the first successful open heart operation [9]. Over the next several decades, the medical community's familiarity with this device and the advancement of the supporting technology led to developments where extracorporeal oxygenation could be attempted on patients outside of the acute operative setting. In 1965, Rashkind et al. [10] were the first to use a bubble oxygenator to support a neonate dying of respiratory failure. By 1975, Bartlett et al. [11] successfully used ECMO in neonates with severe respiratory distress. Since these original pioneers of ECMO, there have been significant advances in the devices and implementations of the artificial lung.

It is important to note that the term ECMO encompasses all of the different components which allow for extracorporeal oxygenation. The ECMO circuit can be broken down into its basic components of vascular access, tubing with inflow from and outflow into the patient, an oxygenator, and any pumps, if needed, to augment the extracorporeal blood flow (Fig. 1). Significant advances over the decades since its inception have been made in these individual areas and will be briefly reviewed below.

\section{Oxygenator}

The oxygenator in which gas exchange takes place has been one of the areas of greatest advancement. In 1953, Gibbon used a stationary film type oxygenator in which oxygen was exposed to a film of blood as it flowed over a series of stainless steel plates. While this direct contact of the blood to the gas did provide the necessary gas exchange, the damage to the blood components was substantial. The development of the bubble oxygenator helped decrease this damage but came with additional issues including increased systemic inflammation and resistance through the ECMO circuit [11]. By 1969, Dorson and colleagues [12] began using a membrane oxygenator which offered the advantage of eliminating the direct contact of the gas with the blood and was considered vastly superior to its predecessors. Over the years, the efficacy of the materials used for the membranes showed significant advancement as well. In the early years of membrane 


\section{A V-V ECMO}

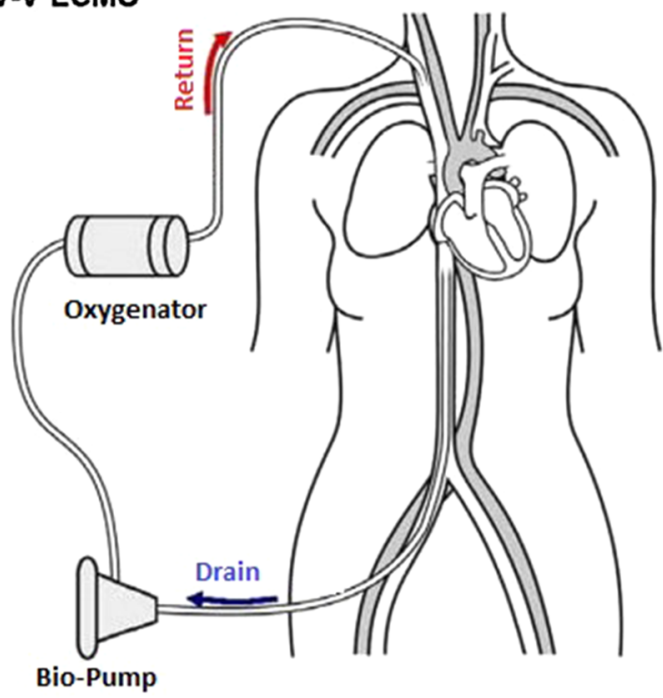

C VV-A ECMO

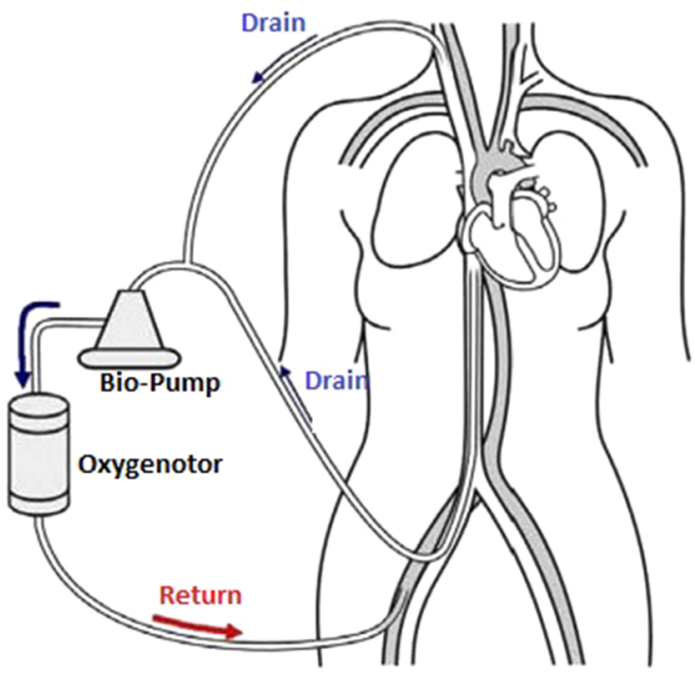

B VVV-A ECMO

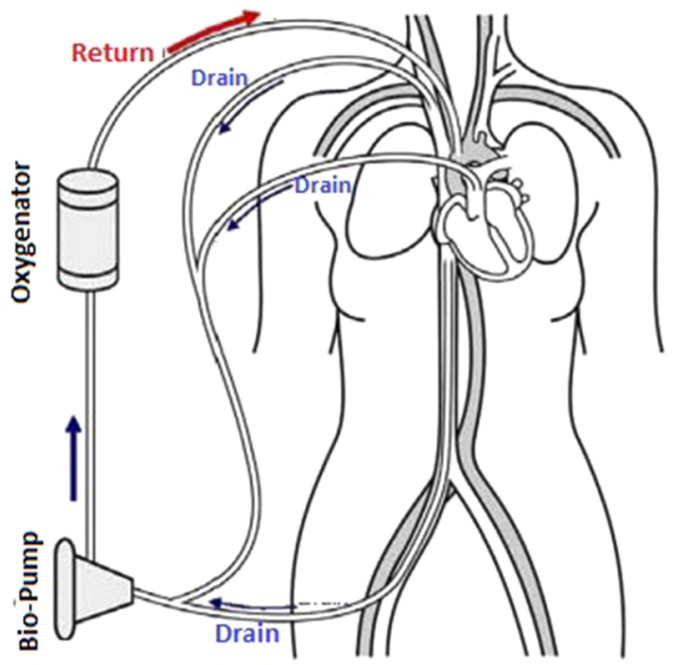

D V-AV ECMO

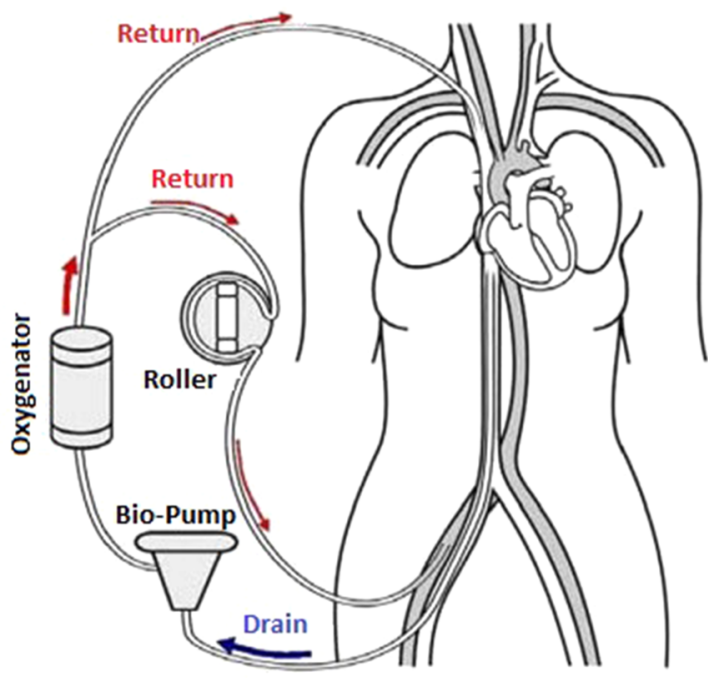

Fig. 1 Components of VV-ECMO Circuit. Tubing connecting the patient, a pump, and an oxygenator with large vessel venous vascular access (http://picsbox.biz/key/vv\%20ecmo\%20circuit)

oxygenation, relatively impermeable polyethylene or Teflon homogeneous membranes were the mainstay [13]. These have since been replaced by silicone rubber membrane oxygenators and the newer polymethylpentene membranes which offer lower resistance, lower consumption of blood products and resulting thrombocytopenia, and offer better gas exchange [14]. Microporus hollow fiber oxygenators, in which gas flows through microfibers around which blood flows, offer some advantages to membrane oxygenators such as lower resistance and higher efficiency, but currently have a limited lifespan, making them less than ideal in a VV-ECMO circuit that may have to last days to weeks.
Vascular Access

Since the origins of VV-ECMO, there has been considerable advancement in the configuration of vascular access. VV-ECMO may be configured in-series or in-parallel (Fig. 2). Each of these options has its own benefits and shortcomings.

As can be appreciated in Fig. 2, the in-parallel configuration has the inlet to the ECMO circuit anastomosed to the pulmonary artery, while the oxygenated outflow is anastomosed to the left atrium. The lower resistance of the ECMO circuit compared to the patient's pulmonary vasculature is what diverts the blood flow away from the 


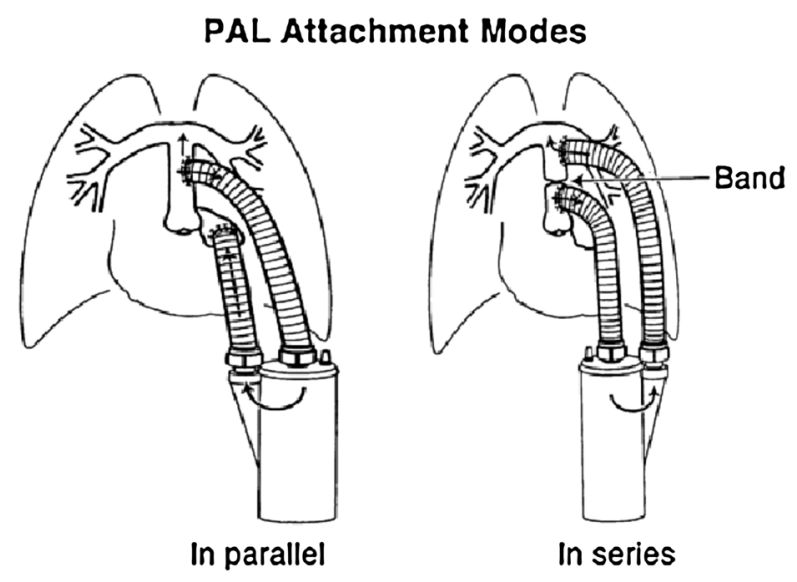

Fig. 2 Traditional Modes of attachment for VV-ECMO. In-parallel inflow from the pulmonary artery with oxygenated outflow returned to the left atrium. In-series inflow attached to the proximal pulmonary artery and outflow attached to the distal pulmonary artery. They pulmonary artery between the two cannulas is ligated. (From Perfusion 2002; 17:253-268; Fig. 4; with permission)

lungs. This makes it an ideal setup for patients that have high pulmonary vascular resistance. In addition to providing oxygenation and ventilation, this setup relieves stress on the right ventricle. The concern with this configuration has been that most of the blood is shunted away from the lungs. Although the reason to provide ECMO is to assist poorly functioning lungs from a respiratory standpoint, the lungs provide many other metabolic and physiologic functions in addition to serving as a mechanical filter. With blood returning directly to the left atrium, there is no filter for the systemic circulation, and any emboli from the patient's venous system or the ECMO circuit itself can cause potential embolization in the form of a stroke, visceral embolization, or extremity ischemia.

The second historically described configuration is the inseries placement. The inflow to the ECMO circuit is once again the pulmonary artery. The outflow back to the patient is anastomosed to the distal pulmonary artery with a restrictive band placed between the inflow and outflow. The advantage of this configuration is that the entire circulation must pass through the pulmonary vasculature, thereby helping maintain the metabolic functions of the lung. At the same time, the lungs can provide protective filtration from any emboli that may originate within the ECMO system. The major downside to this configuration is the large amount of strain that is placed on the right heart [15].

A drawback of the previously mentioned VV-ECMO configurations is that they required surgical implantation into the central vasculature. More recent advances have allowed for percutaneous placement of the venous drainage

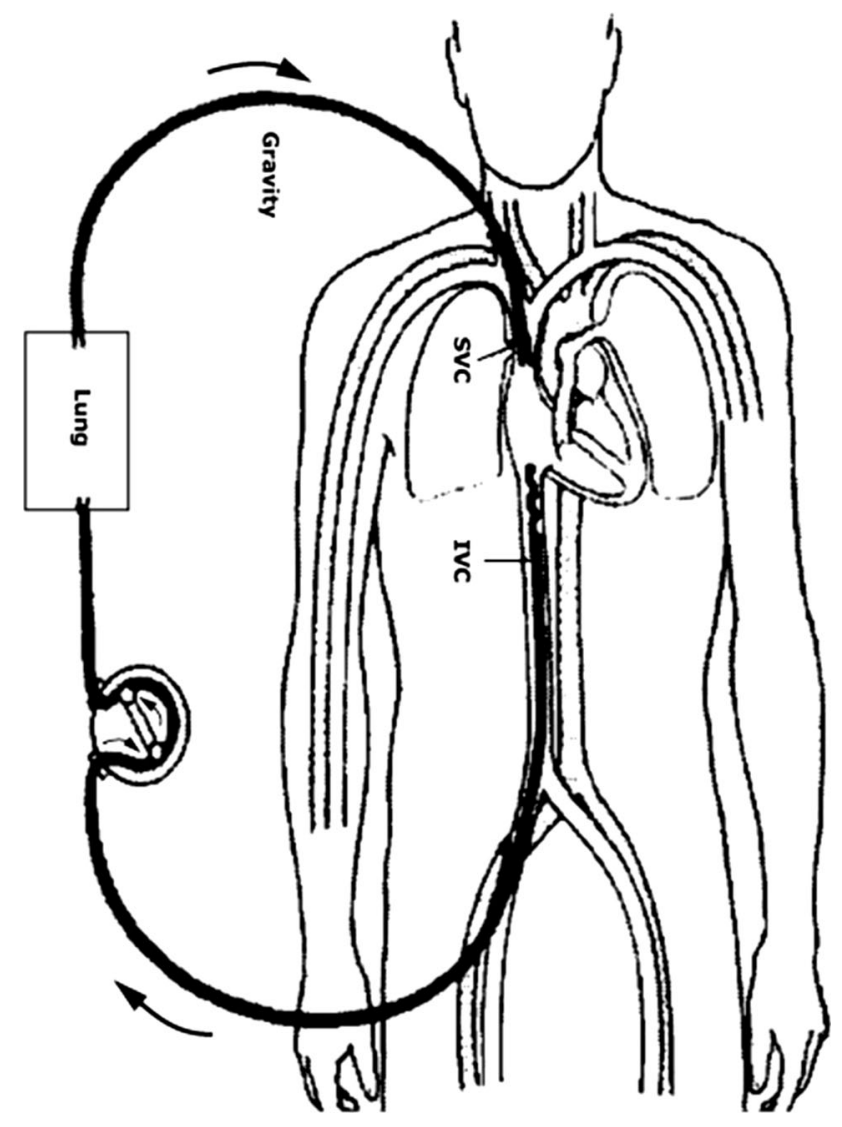

Fig. 3 Veno-venous ECMO configuration in an adult patient using a right common femoral drainage cannula and a right internal jugular return cannula

and return cannulas with fluoroscopic assistance. This may be done with a surgical vessel cut-down approach or via a Seldinger technique. The femoral, internal jugular, or subclavian vessels are the most commonly cannulated vessels for the VV inflow. Oxygenated blood is then usually delivered back to the patient via a cannula positioned in the right atrium. This allows for the advantages experienced with the in-series configuration of VV-ECMO in that the blood passes through the lungs, thereby allowing for mechanical filtration and metabolic activity of the native lungs (Fig. 3).

Over the past several years, a new configuration using a double lumen cannula (DLC) has been developed. With this cannula, as illustrated in Fig. 4, blood inflow to the ECMO circuit is through the inferior vena cava and superior vena cava and returned via an infusion port that directs blood into the right atrium and through the tricuspid valve. Only one access site is required in the right internal jugular vein, making placement relatively straightforward with no central surgical cannulation needed. The major advantage, however, is that with only a single jugular cannula the patient is able to ambulate, while VV-ECMO is being 


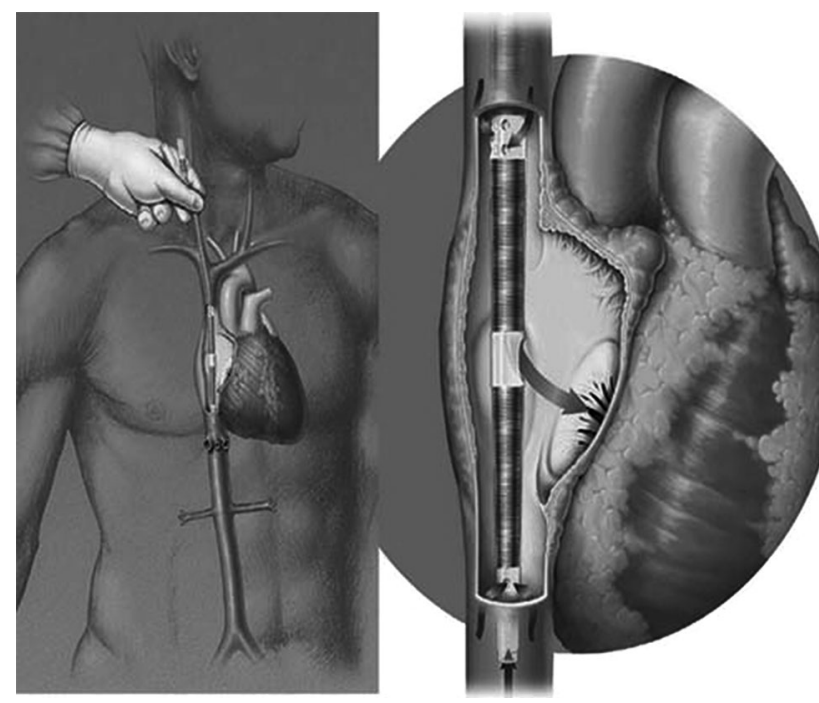

Fig. 4 DLC inserted from the right internal jugular vein. Inflow is obtained from both the inferior vena cave (IVC) and superior vena cava (SVC). Return is at the level of the right atrium and directed across the tricuspid valve. (From ASAIO J 2008; 54:606-611; Fig. 1; with permission)

administered. This helps to reduce the significant morbidity and mortality that chronic bed rest can lead to in the already critically ill population.

\section{The Pump}

In a VV-ECMO circuit, all blood is returned to the venous system and the pump does not support the systemic blood pressure. It does, however, aid in driving the blood through the oxygenator and back to the patient. The three types of pumps most commonly used in VV-ECMO are the roller, centrifugal, and axial pumps. Over the years of advancement, the magnetically driven centrifugal pump has come to the forefront as the most commonly used as it is thought to cause less hemolysis and trauma to the ECMO tubing itself.

\section{Indications for Use}

Each institution may create their own guidelines for the initiation of VV-ECMO based on institution resources and the preferences of the care providers. In the case of acute lung injury, VV-ECMO should only be used in situations of life threatening but potentially reversible lung injury in which the patient is failing conventional ventilator support. The Extracorporeal Life Support Organization (ELSO) sets out guidelines for both hypoxic and hypercarbic indications for ECMO (Fig. 5). In their hypoxic guidelines, ECMO should be considered in patients with respiratory failure due to any cause when risk of mortality exceeds $50 \%$ and should be deployed when the estimated mortality surpasses
$80 \%$. A $50 \%$ mortality risk can be identified by a partial pressure of oxygen in arterial blood $\left(\mathrm{PaO}_{2}\right)$ to the inspired oxygen fraction $\left(\mathrm{FiO}_{2}\right)$ ratio of less than 150 on a $\mathrm{FiO}_{2}$ of greater than $90 \%$ or a Murray Score of 2-3. An $80 \%$ mortality risk can by identified by a $\mathrm{PaO}_{2} / \mathrm{FiO}$ ratio of less than 80 or an $\mathrm{FiO}_{2}$ of greater than $90 \%$ and a Murray score of 3-4. The Murray score is a lung injury severity score that is commonly used which factors in a patient's $\mathrm{PaO}_{2} /$ $\mathrm{FiO}_{2}$ ratio, the required ventilatory positive end-expiratory pressure (PEEP), lung compliance, and X-ray findings. For hypercapnic patients, ELSO guidelines dictate that $\mathrm{CO}_{2}$ retention due to asthma or permissive hypercapnia with a $\mathrm{PaCO}_{2}$ of less than 80 or an inability to achieve safe inflation pressures is an indication for ECMO [16•]. The CESAR trial postulated that a Murray score of 3 or greater or a $\mathrm{pH}$ of less than 7.2 with uncompensated hypercapnia may be used as justification for ECMO [2].

\section{Other Options}

Despite the many advances that have been made, ECMO therapy is not without its inherent complications. Of note by Zangrillo et al. [17], ECMO was complicated by renal failure $(52 \%)$, bacterial pneumonia $(33 \%)$, bleeding (33\%), oxygenator dysfunction (29\%), sepsis (26\%), hemolysis $(18 \%)$, liver dysfunction $(16 \%)$, leg ischemia $(10 \%)$, venous thrombosis $(10 \%)$, central nervous system complications (8\%), gastrointestinal bleeding (7\%), aspiration pneumonia (5\%), and disseminated intravascular coagulation $(5 \%)$. For these reasons, and in the continued quest to produce better, more durable, and potentially intracorporeal artificial lungs devices, other options besides VV-ECMO are under investigation.

One such device is the intravascular oxygenator (IVOX). In this system, a membrane oxygenator which is devised of several hundred gas permeable hollow fibers allows for gas exchange of $\mathrm{O}_{2}$ and $\mathrm{CO}_{2}$. This device is placed into the vena cava via small skin incisions with femoral cannulation. Gas is pumped through these fibers via a vacuum pump or pulsating balloon device. Although this is a novel idea and promising concept, trials to date have not shown that IVOX has been able to reduce a patient's ventilatory requirements and therefore is a long way off from providing those in critical condition with artificial lung function [18].

Another group of lung assist devices is arterio-venous membrane oxygenators. These devices serve as shunts in which blood is driven through an extracorporeal oxygenator by the patients arterio-venous gradient, therefore no pump is needed as in ECMO circuits. Examples of these include the Novalung Interventional Lung Assist device and the pumpless extracorporeal lung assist device ( $\mathrm{pE}$ CLA) $[19,20]$. Since these devices serve as peripheral 
Fig. 5 Indications for VVECMO as suggested by ELSO guidelines $\left[16^{\bullet}\right]$

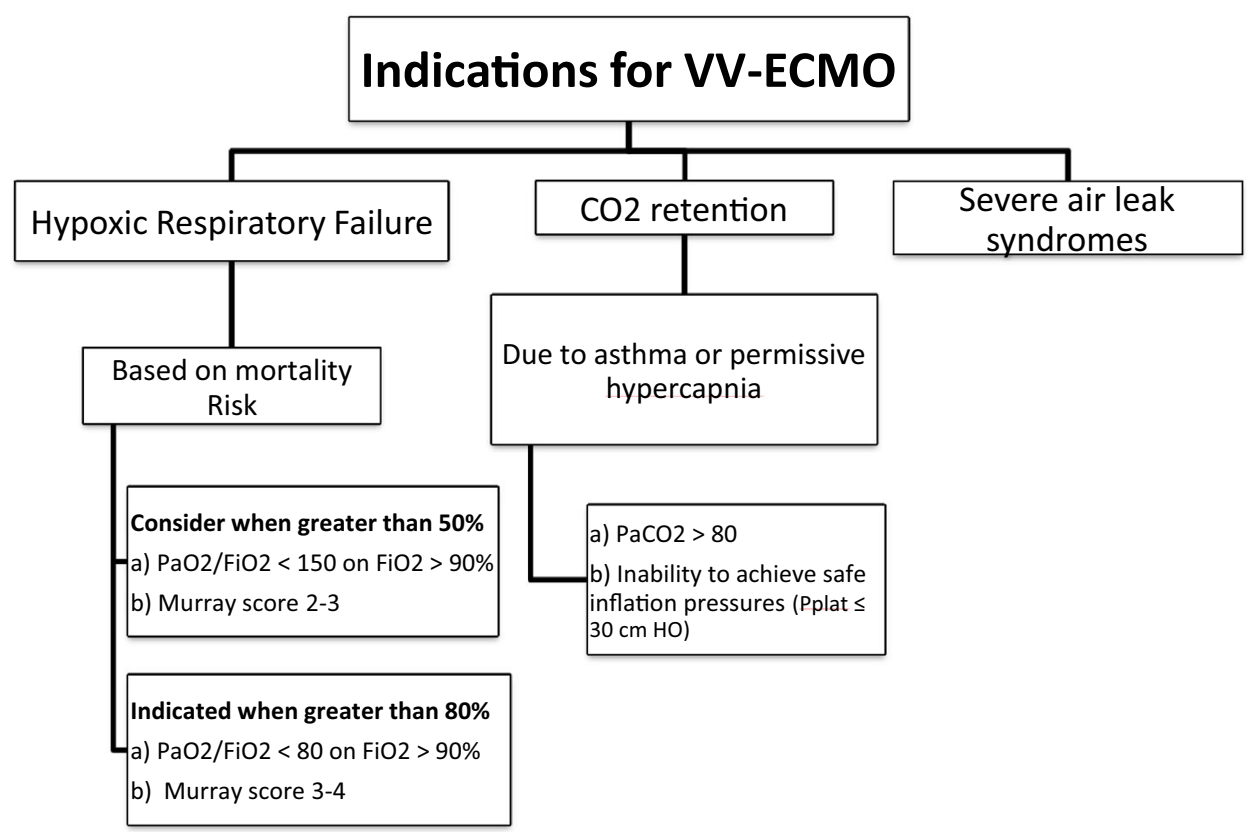

shunts, they have not been found to be as efficient when compared to ECMO circuits. However, without a pump they are easier to manage, making them an attractive option in many situations.

\section{Conclusion and Future}

Recent studies highlighting the benefit of VV-ECMO in patients with significant but reversible lung injury have renewed the medical community's interest in artificial lung technology. These studies have begun to paint a picture that VV-ECMO is a valuable tool in the appropriately selected adult population that can improve our odds in the battle with acute lung injury and in some cases buy much needed time in patients with end-stage lung disease. Our current armamentarium of ECMO technology has dramatically improved since the origins of ECMO in the 1950s. Oxygenators are now smaller and more efficient, pumps are less traumatic to the blood products, and advancement in cannula access has made ambulatory ECMO a reality. There is still tremendous room for progress. Currently, we are limited in the duration of ECMO that can be offered. This limited duration may suffice for patients with acute lung injury in which rapid lung recovery may be obtainable, but more durable and less traumatic systems may allow the benefit of VV-ECMO to an increased segment of the population combating lung failure. The evolution of ambulatory ECMO added the multiple benefits that accompany patient mobility, but patients are still tethered to bulky tubing, oxygenators, and pumps. In the future, these devices may grow ever smaller with the ultimate goal of a completely intracorporeal device. In addition to the artificial lung devices themselves, the parallel advancement of the medical management of patients with lung failure will continue to progress in our quest for the ideal artificial lung.

\section{Compliance with Ethics Guidelines}

Conflict of Interest Nathan L Kister, Brittany A. Zwischenberger, and Jeremiah T. Martin declare no conflict of interest. Joseph B. Zwischenberger reports royalty payments from Maquet (formerly Avalon), as well as research Grants from NIH.

Human and Animal Rights and Informed Consent This article does not contain any studies with human or animal subjects performed by the authors.

\section{References}

Papers of particular interest, published recently, have been highlighted as:

- Of importance

-• Of major importance

1. Vasilyev SS, Chaap RN, Mortensen JD. Hospital survival rates of patients with acute respiratory failure in modern respiratory intensive care units. Chest. 1995;107:1083-8.

2. Zapol WM, Snider MT, Hill JD, et al. Extracorporeal membrane oxygenation in severe acute respiratory failure. A randomized prospective study. MAMA. 1979;242:2193-6.

3. - Peek GJ, Mugford M, Tiruvoipati R, et al. CESAR trial collaboration: efficacy and economic assessment of conventional ventilatory support versus extracorporeal membrane oxygenation for severe adult respiratory failure (CESAR): a multicentre randomised controlled trial. Lancet. 2009;374:1351-63. In this large multi-center trial ECMO was found to have positive results. 
Many of the other references discus the current technology, it's development, and future direction. This is a large trial which shows support for the technology that we already have.

4. Zangrillo A, Biondi-Zoccai G, Landoni G, et al. Extracorporeal membrane oxygenation (ECMO) in patients with $\mathrm{H} 1 \mathrm{~N} 1$ influenza infection: a systematic review and meta-analysis including 8 studies and 266 patients receiving ECMO. Crit Care. 2013;17:R30.

5. •- Martin JT, Zwischenberger JB. Artificial lung and novel devices for respiratory support. Semin Thorac Cardiovasc Surg. 2013;25:70-5. This reference provides a relatively up to date evaluation of current ECMO support devices as well as basic concepts behind the development of artificial lung technology. Lead author Dr. Martin is also a faculty mentor so I was able to discuss all points of the paper in detail.

6. Toyoda Y, Bhama JK, Shigemura N, et al. Efficacy of extracorporeal membrane oxygenation as a bridge to lung transplantation. J Thorac Cardiovasc Surg. 2013;145:1065-70.

7. Orens JB, Garrity ER. General overview of lung transplantation and review of organ allocation. Proc Am Thorac Soc. 2009;6: 13-9.

8. Yeazell L, Littlewood K. Nonrespiratory functions of the lung. In: Slinger P, editor. Principles and practice of anesthesia for thoracic surgery. New York: Springer; 2011. p. 103-19.

9. Gibbon JH Jr. Application of a mechanical heart and lung apparatus to cardiac surgery. Minn Med. 1954;37:180-5.

10. Rashkind WJ, Freeman A, Klein D, Toft RW. Evaluation of a disposable plastic, low volume, pumpless oxygenator as a lung substitute. J Pediatr. 1965;66:94-102.

11. Bartlett RH, Gazzaniga AB, Jefferies MR, et al. Extracorporeal membrane oxygenation (ECMO) cardiopulmonary support in infancy. Trans Am Soc Artif Intern Organs. 1976;22:80-93.

12. Dorson W Jr, Baker E, Cohen ML, Meyer B, Molthan M, Trump D, Elgas R. A perfusion system for infants. Trans Am Soc Artif Intern Organs. 1969;15:155-60.
13. Gaylor JD. Membrane oxygenators: current developments in design and application. J Biomed Eng. 1988;10(6):541-7.

14. Ravindranath T, Botha J, Peek G. Effectiveness of extracorporeal membrane oxygenation when conventional fails: valuable option or vague remedy? J Crit Care. 2012;27:192-8.

15. Nolan H, Wang D, Zwischenberger JB. Artificial lung basics. Fundamental challenges, alternative designs and future innovations. Organogenesis. 2011;7:23-7.

16. - Extracorporeal Life Support Organization. ELSO guidelines for ECMO centers, v1.7. 2010. http://www.elsonet.org/index. php?option=com_phocadownload \& view=category\&id=4\&Itemid= 627. Accessed 15 March 2014. This website was valuable in that it discussed the most up to date recommendations for the use of artificial lung technology.

17. Zangrillo A, Landoni G, Biondi-Zoccai G, et al. A meta-analysis of complications and mortality of extracorporeal membrane oxygenation. Crit Care Resusc. 2013;15:172-8.

18. Gentilello LM, Jurkovich GJ, Gubler KD, Anardi DM, Heiskell $\mathrm{R}$. The intravascular oxygenator (IVOX): preliminary results of a new means of performing extrapulmonary gas exchange. J Trauma. 1993;35:399-404.

19. Bein T, Scherer MN, Philipp A, Weber F, Woertgen C. Pumpless extracorporeal lung Assist (pECLA) in patients with acute respiratory distress syndrome and severe brain injury. J Trauma. 2005;58:1294-7.

20. CTSNet, the Cardiothoracic Surgery Network. The Novalung(R) iLA membrane ventilator: technical aspects. 2012. http:// www.ctsnet.org/portals/thoracic/newtechnology/article-9. Accessed 15 March 2014 